\title{
Asymptotic Disturbance Attenuation Properties for Continuous-Time Uncertain Switched Linear Systems
}

\author{
Hai Lin* Panos J. Antsaklis** \\ * ECE Dept., National University of Singapore, Singapore \\ (e-mail: elelh@nus.edu.sg) \\ ** EE Dept., University of Notre Dame, IN 46556 USA \\ (e-mail: antsaklis.1@nd.edu)
}

\begin{abstract}
In this paper, the disturbance attenuation properties in the sense of uniformly ultimate boundedness are investigated for a class of switched linear systems with parametric uncertainties and exterior disturbances. The aim is to characterize the conditions under which the switched system can achieve a finite disturbance attenuation level. First, arbitrary switching signals are considered, and a necessary and sufficient condition is given. Secondly, conditions on how to restrict the switching signals to achieve finite disturbance attenuation levels are investigated. Two cases are considered here that depend on whether all the subsystems are uniformly ultimately bounded or not. The techniques are based on multiple polyhedral Lyapunov functions and their extensions.
\end{abstract}

\section{INTRODUCTION}

The last decade has seen increasing research activities in the field of switched systems, and the main efforts typically focus on the analysis of dynamic behaviors, such as stability, controllability, reachability, and observability etc. and aim to design controllers with guaranteed stability and optimized performance, see e.g., Liberzon et al. [1999], Decarlo et al. [2000], Hespanha [2003], Sun et al. [2005], Lin et al. [2005, 2007]. However, the literature on robust performance of switched systems is still relatively sparse, and most existing results assume that the disturbances are constrained to have finite energy, i.e., bounded $\mathcal{L}_{2}$ norm, see e.g. Hespanha [2003], Zhai et al. [2001]. In practice, there are disturbances that do not satisfy this condition and act more or less continuously over time. Such disturbances are called persistent, and cannot be treated in the above framework. In this paper, the disturbance attenuation property is in the signal's magnitude sense, i.e., time domain specifications. Moreover, we explicitly consider dynamic uncertainty in the switched system model. Dynamic uncertainty in the plant model is one of the main challenges in control theory, and it is of practical importance to deal with this kind of uncertainties explicitly.

In this paper, we will focus on the disturbance attenuation analysis for classes of switched linear systems which are perturbed by both parameter variations and exterior disturbances. In particular, we consider a collection of continuous-time linear systems described by the perturbed differential equations with parametric uncertainties

$$
\dot{x}(t)=A_{q}(w) x(t)+E_{q} d(t), \quad t \in \mathbb{R}^{+},
$$

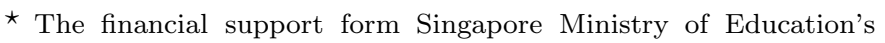
AcRF Tier 1 funding is gratefully acknowledged. where $q \in Q=\left\{q_{1}, q_{2}, \cdots, q_{N}\right\}, \mathbb{R}^{+}$stands for nonnegative reals, the state variable $x(t) \in \mathbb{R}^{n}$, and the disturbance input $d(t) \in \mathcal{D} \subset \mathbb{R}^{r}$. Assume that $\mathcal{D}$ is a $\mathrm{C}$-set. The term $\mathrm{C}$-set stands for a convex and compact set containing the origin in its interior. Assume polytopic uncertainties in (1), i.e., $A_{q}(w)=\sum_{j=1}^{v_{q}} w_{j} A_{q}^{j}, w_{j} \geq 0$ and $\sum_{j=1}^{v_{q}} w_{j}=1$. Notice that $A_{q}^{j}$ are known constant $n \times n$ matrices and the coefficients $w_{j}$ are unknown and possibly time varying. Without loss of generality, we assume that $E_{q} \in \mathbb{R}^{n \times r}$ is a constant matrix.

Combine the family of continuous-time uncertain linear systems (1) with a class of piecewise constant functions, $\sigma$ : $\mathbb{R}^{+} \rightarrow Q$. Then we can define the following time-varying system as a continuous-time switched linear system

$$
\dot{x}(t)=A_{\sigma(t)}(w) x(t)+E_{\sigma(t)} d(t), \quad t \in \mathbb{R}^{+},
$$

where $\sigma(t)$ is called a switching signal.

It is known that the dynamic properties of a switched system depend not only on its subsystems but also on the switching signals. The aim of this paper is to characterize the dynamics of each subsystem and the properties of switching signals such that the switched systems generate convergent behaviors. Because of parameter variations and exterior disturbances, it is only reasonable to expect that the trajectories of the switched system converge into a neighborhood region of the equilibrium (the origin here) in the following sense.

Definition 1. The uncertain switched system (2) under switching signal $\sigma(t)$ is Uniformly Ultimately Bounded $(U U B)$ if there exists a $\mathrm{C}$-set $\mathcal{S}$ such that for every initial condition $x(0)=x_{0}$, there exists a finite $T\left(x_{0}\right)$, and $x(t) \in \mathcal{S}$ for $t \geq T\left(x_{0}\right)$.

The disturbance attenuation properties considered here are in the sense of the uniformly ultimate boundedness. Given a collection of switching signals, if the switched 
system (2) is UUB for all these switching signals, then the switched system (2) is said to have a finite disturbance attenuation level under this class of switching signals. If the switched system is UUB for all possible switching signals, the switched system (2) is said to a have finite disturbance attenuation level under arbitrary switchings. We are also interested in characterizing a useful subclass of switching signals such that the switched system (2) achieves a finite disturbance attenuation level even when some of its subsystems do not have finite disturbance attenuation levels.

The rest of the paper is organized as follows. In Section 2, the disturbance attenuation properties under arbitrary switching signals are considered. The aim is to identify necessary and sufficient conditions under which the switched system preserves uniformly ultimate boundedness under arbitrary switching signals. Secondly, when the finite disturbance attenuation level is not preserved under arbitrary switching signals, we turn to characterize the subclasses of switching signals so as to guarantee the achievement of a finite disturbance attenuation level. Two cases are considered for this problem that depend on whether all the subsystems are UUB or not, which are developed in Section 3 and Section 4 respectively.

Notation: The letters $\mathcal{E}, \mathcal{P}, \mathcal{S} \cdots$ denote sets, and $\partial \mathcal{P}$ the boundary of set $\mathcal{P}$. For any real $\lambda \geq 0$, the set $\lambda \mathcal{S}$ is defined as $\{x=\lambda y, y \in \mathcal{S}\}$. A polytope (bounded polyhedral set) $\mathcal{P}$ will be presented either by a set of linear inequalities $\mathcal{P}=\left\{x: F_{i} x \leq g_{i}, i=1, \cdots, s\right\}$, or by the dual representation in terms of the convex hull of its vertex set $\left\{x_{j}\right\}$, denoted by $\operatorname{Conv}\left\{x_{j}\right\}$.

\section{PERFORMANCE UNDER ARBITRARY SWITCHINGS}

When there is no restriction or a priori knowledge on the switching signals, arbitrary switching are usually assumed. In addition, in the multiple-controller design, see e.g. Hespanha et al. [2002], it is often desirable to guarantee the stability under all possible switching among these controllers, which may provide more flexibility in operation and hence achieve better performances. Therefore, we consider arbitrary switching in this section and derive necessary and sufficient conditions for the switched systems to achieve finite disturbance attenuation properties. For this, it is necessary to require that every subsystem has a finite disturbance attenuation level. However, even when all the subsystems of a switched system are UUB, it is still possible to construct a divergent trajectory from any initial state for such a switched system. The interesting question is under what condition (necessary and sufficient) the switched linear system (2) achieves a finite asymptotic disturbance attenuation level.

For such a purpose, let us first recall a result from the robust stability literature, see e.g. Molchanov et al. [1989], for the following linear time-variant system

$$
\dot{x}(t)=A(t) x(t)
$$

where $A(t) \in \mathcal{A} \hat{=} \operatorname{Conv}\left\{A_{q_{1}}^{1}, A_{q_{1}}^{2}, \cdots, A_{q_{1}}^{v_{q_{1}}}, A_{q_{2}}^{1}, \cdots, A_{q_{2}}^{v_{q_{2}}}\right.$, $\left.\cdots, A_{q_{N}}^{1}, \cdots, A_{q_{N}}^{v_{q_{N}}}\right\}$. In other words, the state matrix $A(t)$ of the above linear time-variant system (3) is constructed by a convex combinations (with time-variant coefficients) of all the subsystems' vertex matrices of the switched linear system (2). Let us denote the index set for these vertices as I. A necessary and sufficient condition for the asymptotic stability of the above linear time-variant system (3) is stated as follows.

Lemma 1. (Molchanov et al. [1989]) The linear timevariant system (3) is asymptotically stable if and only if there exist a full column rank matrix $M \in \mathbb{R}^{m \times n}, m \geq n$, and a family of matrices $\left\{\bar{A}_{i} \in \mathbb{R}^{m \times m}: i \in \mathfrak{I}\right\}$ with strictly negative row dominating diagonal, such that the matrix relations

$$
M A_{i}=\bar{A}_{i} M
$$

are satisfied.

As an immediate consequence, a necessary and sufficient condition for the switched systems (2) with a finite asymptotic disturbance attenuation level under arbitrary switching signals can be expressed as the following proposition.

Proposition 2. The following statements are equivalent:

(1) The switched linear system (2) achieves a finite asymptotic disturbance attenuation level under arbitrary switching;

(2) The undisturbed switched linear system

$$
\dot{x}(t)=A_{\sigma(t)}(w) x(t),
$$

where $A_{\sigma(t)}(w) \in\left\{A_{q_{1}}(w), A_{q_{2}}(w), \cdots, A_{q_{N}}(w)\right\}$, is globally asymptotically stable under arbitrary switching;

(3) the linear time-variant system (3) is asymptotically stable;

(4) there exists a full column rank matrix $M \in \mathbb{R}^{m \times n}$, $m \geq n$, such that for any $A_{i} \in \operatorname{vert}\{\mathcal{A}\}$ the matrix relation

$$
M A_{i}=\bar{A}_{i} M
$$

holds for some matrix $\bar{A}_{i} \in \mathbb{R}^{m \times m}$ with strictly negative row dominating diagonal.

It is interesting to notice that the robust stability of a polytopic uncertain linear time-variant system, which has infinite number of possible dynamics (modes), is equivalent to only considering a finite number of its vertex dynamics as an arbitrary switching system. This fact bridges two originally distinct research fields. Based on the equivalence between the asymptotic stability of arbitrary switching linear systems and the robust stability of polytopic uncertain linear time varying systems, some well established converse Lyapunov theorems can be introduced for arbitrary switching linear systems. For example, the following result was adopted from (Molchanov et al. [1989]).

Theorem 3. If a switched linear system (2) is asymptotically stable under arbitrary switching signals, then there exists a polyhedral Lyapunov function, which is monotonically decreasing along the switched linear system's trajectories.

Compared with the existing converse Lyapunov theorems, e.g. Dayawansa et al. [1999], the above result has the following advantages. First, it shows that one may solely focus on polyhedral Lyapunov function without loss of generality. Second, there exists automated computational methods to calculate polyhedral Lyapunov functions, see e.g. Brayton et al. [1979], Blanchini [1995], Polanski [2000], Yfoulis et al. [2004]. In the next sections, we will employ 
multiple polyhedral Lyapunov functions and their extensions to study the stability issues for the switched systems under constrained, instead of arbitrary, switching signals.

\section{PERFORMANCE UNDER SLOW SWITCHING: UUB SUBSYSTEMS}

If the finite disturbance attenuation level is not preserved under arbitrary switching signals, it is still possible to restrict the switching signals so as to achieve a finite disturbance attenuation level. Therefore, it is interesting to classify the classes of switching signals under which the switched system remains UUB.

\subsection{Set-Induced Lyapunov Function}

Following the notation of Blanchini [1994], we call a function $\Psi: \mathbb{R}^{n} \rightarrow \mathbb{R}$ a gauge function if $\Psi(x) \geq 0$, $\Psi(x)=0 \Leftrightarrow x=0$; for $\mu>0, \Psi(\mu x)=\mu \Psi(x)$; and $\Psi(x+y) \leq \Psi(x)+\Psi(y), \forall x, y \in \mathbb{R}^{n}$. A gauge function is convex and it defines a distance of $x$ from the origin which is linear in any direction. If $\Psi$ is a gauge function, we define the closed set (possibly empty) $\bar{N}[\Psi, \xi]=\left\{x \in \mathbb{R}^{n}\right.$ : $\Psi(x) \leq \xi\}$. It is easy to show that the set $N[\Psi, \xi]$ is a Cset for all $\xi>0$. On the other hand, any $\mathrm{C}$-set $\mathcal{S}$ induces a gauge function $\Psi_{\mathcal{S}}(x)$ (Known as Minkowski function of $\mathcal{S})$, which is defined as $\Psi(x) \doteq \inf \{\mu>0: x \in \mu \mathcal{S}\}$. Therefore a $\mathrm{C}$-set $\mathcal{S}$ can be thought of as the unit ball $\mathcal{S}=\bar{N}[\Psi, 1]$ of a gauge function $\Psi$ and $x \in \mathcal{S} \Leftrightarrow \Psi(x) \leq 1$.

Consider the continuous-time subsystem

$$
\dot{x}(t)=A_{q}(w) x(t)+E_{q} d(t)
$$

and assume it is UUB along with a Lyapunov function in the following sense.

Definition 4. Given a C-set $\mathcal{S}$, a Lyapunov function outside $\mathcal{S}$ for the subsystem (4) is defined as a continuous function $\Psi_{q}: \mathbb{R}^{n} \rightarrow \mathbb{R}^{+}$such that $\bar{N}\left[\Psi_{q}, \kappa\right] \subset \mathcal{S}$, for some positive scalar $\kappa$, for which the following condition holds: if $x \notin \bar{N}[\Psi, \kappa]$ then $\exists \beta_{q}>0$ such that

$$
\mathcal{D}^{+} \Psi_{q}(x(t)) \leq-\beta_{q} \Psi_{q}(x(t))
$$

Here $\mathcal{D}^{+} \Psi_{q}(x(t))$ stands for the upper right Dini derivative of $\Psi_{q}(x(t))$ along the trajectories of system (4), which is defined as

$$
\mathcal{D}^{+} \Psi_{q}(x(t))=\lim \sup _{\tau \rightarrow 0^{+}} \frac{\Psi_{q}(x(t+\tau))-\Psi_{q}(x(t))}{\tau} .
$$

Under the assumption that $d(t)$ and $w(t)$ are continuous, then the value of the Dini derivative of the point $x(t)=x$ equals

$\mathcal{D}^{+} \Psi_{q}(x(t))=\lim \sup _{\tau \rightarrow 0^{+}} \frac{\Psi_{q}\left(x+\tau\left[A_{q}(w) x+E_{q} d\right]\right)-\Psi_{q}(x)}{\tau}$

where $x(t)=x, d(t)=d$ and $w(t)=w$ (Blanchini [1995]).

Based on differential inequality theory, it can be derived from the above Lyapunov function definition that

$$
\Psi_{q}(x(t)) \leq \max \left\{e^{-\beta_{q}\left(t-t_{0}\right)} \Psi_{q}\left(x\left(t_{0}\right)\right), \kappa\right\}, \quad \forall t>t_{0},
$$

for a trajectory $x(t)$ of (4) starting from $x\left(t_{0}\right)$ at time $t_{0}$. This further implies that (4) is UUB in $\mathcal{S}$. In addition, it was shown in Blanchini [1995] that if a Lyapunov function exists that solves the uniform ultimate boundedness problem in a certain convex neighborhood of the origin for system (4), then there exists a polyhedral Lyapunov function that solves the problem in the same neighborhood. Therefore, without loss of generality, we assume that each subsystem (4) has a polyhedral Lyapunov function $\Psi_{q}$ outside $\mathcal{S}$.

The next question is how to compute the polyhedral Lyapunov function $\Psi_{q}(\cdot)$. To this aim, we consider the Euler approximating system (EAS) of (4) as the following discrete-time system:

$$
x(t+1)=\left[I+\tau A_{q}(w)\right] x(t)+\tau E_{q} d(t)
$$

The connection between the EAS (8) and its original continuous-time system (4) is through the concept of contractive set which is defined as follows.

Definition 5. Given $\lambda, 0<\lambda<1$, a set $\mathcal{S}$ is said $\lambda$ contractive with respect to the $\operatorname{EAS}(8)$, if for any $x \in \mathcal{S}$, $\operatorname{post}_{1}(x, \mathcal{W}, \mathcal{D}) \subseteq \lambda \mathcal{S}$. Here $\operatorname{post}_{q}(\cdot)$ is defined as

$$
\begin{aligned}
& \operatorname{post}_{q}(x, \mathcal{W}, \mathcal{D}) \\
& =\left\{x^{\prime}: x^{\prime}=\left[I+\tau A_{q}(w)\right] x+E_{q} d ; \forall w \in \mathcal{W}, d \in \mathcal{D}\right\}
\end{aligned}
$$

which represents all the possible next step states of (8) from the current state $x$.

The calculation of a $\lambda$-contractive set can be achieved through the following iterative procedure (Blanchini [1994]).

Let $\mathcal{S}$ be an assigned C-set in $\mathbb{R}^{n}$. Consider the following sequence of sets:

$$
\left\{\mathcal{X}_{k}\right\}: \mathcal{X}_{0}=\mathcal{S}, \quad \mathcal{X}_{k}=\operatorname{pre}_{q}\left(\lambda \mathcal{X}_{k-1}\right) \cap \mathcal{S} ; \quad k=1,2, \cdots
$$

where $\operatorname{pre}_{q}(\mathcal{S})$ is defined as

$$
\operatorname{pre}_{q}(\mathcal{S})=\left\{x \in \mathbb{R}^{n}: \operatorname{post}_{q}(x, \mathcal{W}, \mathcal{D}) \subseteq \mathcal{S}\right\}
$$

Once the above procedure terminates, in the sense of $\mathcal{X}_{k+1}=\mathcal{X}_{k}$ (assume non-empty), then it returns a $\lambda$ contractive set contained in $\mathcal{S}$. Actually, the obtained set is the maximal $\lambda$-contractive subset in $\mathcal{S}$. We say that a $\lambda$-contractive set $\mathcal{P}_{m} \subseteq \mathcal{S}$ is maximal in $\mathcal{S}$ if and only if every $\lambda$-contractive set $\mathcal{P}$ contained in $\mathcal{S}$ is also contained in $\mathcal{P}_{m}$.

Proposition 6. If the above procedure (9) terminates and returns an nonempty set, denoted as $\mathcal{P}_{\lambda}$, for some $0<$ $\lambda<1$ and $\tau>0$, then the system (4) is uniformly ultimately bounded (UUB) in $\mathcal{P}_{\lambda} \subseteq \mathcal{S}$. In addition, $\mathcal{P}_{\lambda}$ is a C-set, whose induced Minkowski functional $\Psi_{\mathcal{P}_{\lambda}}(x)$ serves as a Lyapunov function for (4) outside $\mathcal{S}$. In particular, $N\left[\Psi_{\mathcal{P}_{\lambda}}, 1\right] \subset \mathcal{S}$, and for $x \notin N[\Psi, 1]$

$$
\mathcal{D}^{+} \Psi_{\mathcal{P}_{\lambda}}(x(t)) \leq-\frac{1-\lambda}{\tau} \Psi_{\mathcal{P}_{\lambda}}(x(t)) .
$$

The proof of this proposition can be found in Blanchini [1994, 1995]. Such Lyapunov function is uniquely generated from the target set $\mathcal{S}$ for any fixed $\lambda$, so it is named Set-induced Lyapunov Function (SILF) in the literature, see Blanchini [1994, 1995] and its references. For systems with linearly constrained uncertainties, it can be shown that such function may be derived by numerically efficient algorithms involving polyhedral sets, see e.g. Polanski [2000]. 


\subsection{UUB Analysis}

It is assumed that each subsystem is UUB with a decay rate $\lambda_{q}$ along with a polyhedral Lyapunov function, $\Psi_{q}(\cdot)$. Now, define the multiple Lyapunov function candidate as

$$
V(x(t))=\Psi_{\sigma(t)}(x(t))
$$

Let $t_{1}, t_{2}, \cdots$ stand for the time points at which switching occurs, and write $q_{j}$ for the value of $\sigma(t)$ on $\left[t_{j-1}, t_{j}\right)$. Then, for any $t$ satisfying $t_{0}=0<\cdots<t_{i} \leq t<t_{i+1}$, we obtain

$$
V(x(t)) \leq \max \left\{e^{-\beta_{q_{i}}\left(t-t_{i}\right)} \Psi_{q_{i}}\left(x\left(t_{i}\right)\right), 1\right\}
$$

Also, there exists a constant scalar $\mu$ such that $\Psi_{q_{i}}(x) \leq$ $\mu \Psi_{q_{j}}(x)$ and $\Psi_{q_{j}}(x) \leq \mu \Psi_{q_{i}}(x)$, for all $x \in \mathbb{R}^{n}$. A possible choice for $\mu$ is the largest value among $\Psi_{j}\left(v_{j}\right), \forall v_{j} \in$ $\operatorname{vert}\left\{\bar{N}\left[\Psi_{i}, 1\right]\right\}$, and $\Psi_{i}\left(v_{j}\right), \forall v_{j} \in \operatorname{vert}\left\{\bar{N}\left[\Psi_{j}, 1\right]\right\}$. This can be verified by exploring the geometric property of the level sets of $\Psi_{i}(x)$ and $\Psi_{j}(x)$, which is omitted due to space limit.

Denote $\beta_{0}=\min _{q \in Q}\left\{\beta_{q}\right\}$. Then

$$
\begin{aligned}
V(x(t)) & \leq \max \left\{e^{-\beta_{q_{i}}\left(t-t_{i}\right)} \Psi_{q_{i}}\left(x\left(t_{i}\right)\right), 1\right\} \\
& \leq \max \left\{e^{-\beta_{0}\left(t-t_{i}\right)} \Psi_{q_{i}}\left(x\left(t_{i}\right)\right), 1\right\} \\
& \leq \max \left\{e^{-\beta_{0}\left(t-t_{i}\right)} \mu \Psi_{q_{i-1}}\left(x\left(t_{i}\right)\right), 1\right\} \\
& \leq \max \left\{e^{-\beta_{0}\left(t-t_{i}\right)} \mu e^{-\beta_{0}\left(t_{i}-t_{i-1}\right)} \Psi_{q_{i-1}}\left(x\left(t_{i-1}\right)\right), 1\right\} \\
& \leq \cdots \\
& \leq \max \left\{e^{-\beta_{0} t} \mu^{i} \Psi_{q_{0}}(x(0)), 1\right\} \\
& =\max \left\{e^{-\beta_{0} t} \mu^{N_{\sigma}(t)} V(x(0)), 1\right\}
\end{aligned}
$$

where $N_{\sigma}(t)$ denotes the number of switchings of $\sigma(t)$ over the interval $[0, t)$. Assume that there exists a scalar $0<\beta^{*}<\beta_{0}$ such that

$$
e^{-\beta_{0} t} \mu^{N_{\sigma}(t)} \leq e^{-\beta^{*} t}
$$

This inequality is equivalent to

$$
N_{\sigma}(t) \leq \frac{t}{\tau_{a}^{*}}, \quad \tau_{a}^{*}=\frac{\ln \mu}{\beta_{0}-\beta^{*}},
$$

which is exactly an average dwell time scheme. The constant $\tau_{a}^{*}$ is called the average dwell time. The idea is that there may exist consecutive switching separated by less than $\tau_{a}^{*}$, but the average time interval between consecutive switchings is not less than $\tau_{a}^{*}$. Note that the concept of average dwell time between subsystems was originally proposed in Hespanha et al. [1999], and was extended to the discrete-time case in Zhai et al. [2002].

Then we obtain $V(x(t)) \leq \max \left\{e^{-\beta^{*} t} V(x(0)), 1\right\}$, which implies that the switched system is UUB. In summary, we have

Theorem \%. If all the subsystems of switched system (2) are UUB, then the switched system (2) achieves a finite asymptotic disturbance attenuation level under switching signals with an average dwell time no less than $\tau_{a}^{*}=\frac{\ln \mu}{\beta_{0}-\beta^{*}}$ in the sense of (12).

\section{PERFORMANCE UNDER SLOW SWITCHING: WITH NON-UUB SUBSYSTEMS}

In the previous section, we specified a class of slow switching signals that guarantee the uniformly ultimate boundedness for uncertain switched linear systems (2) with stable subsystems. However, there are some cases that it is unavoidable to switch to unstable subsystems, such as controller failure in fault tolerant systems, packet dropouts in networked control systems etc.

In this section, we will study the case when not all the subsystems are uniformly ultimately bounded. Without loss of generality, it is assumed that the first $r$ subsystem are UUB along with a Lyapunov function, while the rest of the subsystems are not UUB. To make the problem tractable, the expansion rates of these unstable subsystems are limited. In particular, we assume that the expansion of the unstable subsystems are bounded in the sense of the existence of a set-induced Lyapunov-like function, which is introduced below and represents an extension of the classical set-induced Lyapunov functions.

\subsection{Set-Induced Lyapunov-like Functions}

For the subsystem that is not UUB, there does not exist set-induced Lyapunov function as derived in the previous section. Therefore, we generalize the concept of $\lambda$-contractive set and derive a set-induced Lyapunovlike function. For such a purpose, we first introduce the following definition for a Lyapunov-like function.

Definition 8. A Lyapunov-like function outside $\mathcal{S}$ for the continuous-time subsystem (1) can be defined as a continuous function $\Psi_{q}: \mathbb{R}^{n} \rightarrow \mathbb{R}^{+}$such that $\bar{N}\left[\Psi_{q}, \kappa\right] \subset \mathcal{S}$, for some positive scalar $\kappa$, for which the following condition holds: if $x \notin \bar{N}\left[\Psi_{q}, \kappa\right]$ then there exists $\beta_{q}>0$ such that $\mathcal{D}^{+} \Psi_{q}(x(t)) \leq \beta_{q} \Psi_{q}(x(t))$.

This Lyapunov-like function outside $\mathcal{S}$ is quite similar to the definition of a Lyapunov function outside $\mathcal{S}$ in the previous section. The difference is that the value of a Lyapunov-like function may increase along state trajectories instead of decreasing. To capture this trend of expansion in the state space, we introduce the following definition of an expansive set, which is a counter-part to the contractive set defined in the previous section.

Definition 9. Given $\lambda, \lambda>1$, a set $\mathcal{S}$ is said to be $\lambda$ expansive with respect to the $\operatorname{EAS}(8)$, if for any $x \in \mathcal{S}$ then $\operatorname{post}_{q}(x, \mathcal{W}, \mathcal{D}) \subseteq \lambda \mathcal{S}$.

The purpose to introduce the concept of the expansive set for an unstable subsystem's EAS (8) is to determine a Lyapunov-like function for this unstable subsystem (4) so as to capture its explosiveness.

Definition 10. The EAS (8) is said to have an expansive index $\lambda>1$ to the $\mathrm{C}$-set $\mathcal{S}$ if there exists a gauge function $\Psi(x)$ and a constant $\xi>0$ such that the ball $\bar{N}[\Psi, \xi] \subseteq \mathcal{S}$ and, if $x \notin \operatorname{int}\{\bar{N}[\Psi, \xi]\}$, then $\Psi\left(\right.$ post $\left._{q}(x, w, d)\right) \leq \lambda \Psi(x)$ for all $w \in \mathcal{W}$ and $d \in \mathcal{D}$ (or. equivalently, $\bar{N}[\Psi, \mu]$ is $\lambda$-expansive for all $\mu \geq \xi$ ).

Intuitively, the concepts of $\lambda$-expansive set and expansive index $\lambda$ reflect how explosive the unstable subsystems are. For LTI subsystems, this is related to the magnitude of 
their unstable eigenvalues. It is straightforward to show that a $\lambda$-expansive set has the following property.

Lemma 2. If $\mathcal{P}$ is $\lambda$-expansive set for the $\operatorname{EAS}(8)$, then $\mu \mathcal{P}$ is so for all $\mu \geq 1$. (if $\mathcal{D}=\{0\}$, for all $\mu \geq 0$.)

Proof: Let $x \in \mu \mathcal{P}$, hence $\mu^{-1} x \in \mathcal{P}$, so post $\left.\operatorname{pos}_{q}^{-1} x, \mathcal{W}, \mathcal{D}\right)$ $\subset \lambda \mathcal{P}$. Note $\mu^{-1} \mathcal{D} \subset \mathcal{D}$, so $\operatorname{post}_{q}(x, \mathcal{W}, \mathcal{D})=\mu \times$ post $_{q}\left(\mu^{-1} x, \mathcal{W}, \mu^{-1} \mathcal{D}\right) \subset \mu \times$ post $_{q}\left(\mu^{-1} x, \mathcal{W}, \mathcal{D}\right) \subset \lambda \mu \mathcal{P}$.

The next question is how to determine such a $\lambda$-expansive set for an unstable subsystem. It turns out that the procedure developed for the contractive sets (9) can be extended to the case of expansive set in parallel. Similarly, a $\lambda$-expansive set $\mathcal{P}_{\lambda} \subseteq \mathcal{S}$ is maximal in $\mathcal{S}$ if and only if every $\lambda$-expansive set $\mathcal{P}$ contained in $\mathcal{S}$ is also contained in $\mathcal{P}_{\lambda}$.

Proposition 11. The maximal $\lambda$-expansive set $\mathcal{P}_{\lambda} \subseteq \mathcal{S}$ is given by $\mathcal{P}_{\lambda}=\bigcap_{k=0}^{\infty} \mathcal{X}_{k}$.

Proof : First, we show that $\mathcal{X}_{k+1} \subset \mathcal{X}_{k}$. Indeed, $\mathcal{X}_{1} \subset \mathcal{X}_{0}$. Assume that $\mathcal{X}_{k} \subset \mathcal{X}_{k-1}$ then $\lambda \mathcal{X}_{k} \subset \lambda \mathcal{X}_{k-1}$, so $\mathcal{X}_{k+1}=$ $\operatorname{pre}_{q}\left(\lambda \mathcal{X}_{k}\right) \cap \mathcal{S} \subset \operatorname{pre}_{q}\left(\lambda \mathcal{X}_{k-1}\right) \cap \mathcal{S}=\mathcal{X}_{k}$.

Next, we prove that if $\mathcal{P}_{\lambda}$ is nonempty, then it is $\lambda$ expansive. If $x \in \mathcal{P}_{\lambda}$, then $x \in \mathcal{X}_{k}$ for all $k$. For $h \geq 0$ and $k \geq h, \operatorname{post}_{q}(x, \mathcal{W}, \mathcal{D}) \subset \lambda \mathcal{X}_{k-1} \subset \lambda \mathcal{X}_{h-1}$. For all $w \in \mathcal{W}$ and $d \in \mathcal{D}, \operatorname{post}_{q}(x, w, d) \in \lambda \mathcal{X}_{h-1}$. Since $h$ is arbitrary, so post $_{q}(x, w, d) \in \lambda \mathcal{P}_{\lambda}$. Therefore, $\mathcal{P}_{\lambda}$ is $\lambda$-expansive.

Finally, we prove that $\mathcal{P}_{\lambda}$ is maximal. Let $\mathcal{P} \subset \mathcal{X}_{0}$ be $\lambda$-expansive. Assume $\mathcal{P} \subset \mathcal{X}_{k}$. For any $x \in \mathcal{P}$, post $_{q}(x, \mathcal{W}, \mathcal{D}) \subset \lambda \mathcal{P} \subset \lambda \mathcal{X}_{k}$. Hence, $x \in \mathcal{X}_{k+1}$, then $\mathcal{P} \subset \mathcal{X}_{k+1}$. Therefore, $\mathcal{P} \subset \mathcal{X}_{k}$ for all $k$. Thus, $\mathcal{P} \subset \mathcal{P}_{\lambda}$. $\square$

The above iterative procedure for determining the maximal $\lambda$-expansive set may fail to terminate in finite steps. However, under certain conditions, the maximal $\lambda$ expansive set $\mathcal{P}_{\lambda}$ could be determined by finite iterations as shown below.

Proposition 12. Assume that $\mathcal{P}_{\lambda}$ is a C-set, and $\lambda>1$. Then for every $\lambda^{*}$ such that $1<\lambda<\lambda^{*}$, there exists $\mathbf{k}$ such that $\mathcal{X}_{k}$ is $\lambda^{*}$-expansive for all $k \geq \mathbf{k}$.

Proof : Let $\xi=\frac{\lambda^{*}}{\lambda}>1$. There exists $\mathbf{k}$ such that $\mathcal{P}_{\lambda} \subset \mathcal{X}_{k} \subset \xi \mathcal{P}_{\lambda}$, for $k \geq \mathbf{k}$. Since $\xi \mathcal{P}_{\lambda}$ is $\lambda$-expansive (from Lemma 2), for any $x \in \mathcal{X}_{k}$, so $x \in \xi \mathcal{P}_{\lambda}$, and $\operatorname{post}_{q}(x, \mathcal{W}, \mathcal{D}) \subset \lambda \xi \mathcal{P}_{\lambda} \subset \lambda \xi \mathcal{X}_{k}=\lambda^{*} \mathcal{X}_{k}$.

With the existence and determination of a non-empty maximal $\lambda$-expansive set $\mathcal{P}_{\lambda} \subseteq \mathcal{S}$ for the EAS (8), we may induce a Lyapunov-like function from $\mathcal{P}_{\lambda}$ for the continuous-time system (4) as the following result implies.

Proposition 13. Given a C-set $\mathcal{S}$, if the procedure (9) terminates and returns a nonempty $\mathrm{C}$-set $\mathcal{P}_{\lambda}$ for some $\lambda>1$ and $\tau>0$ for (8), then the Minkowski function of $\mathcal{P}_{\lambda}, \Psi_{\mathcal{P}_{\lambda}}(x)$, is a Lyapunov-like function for the system (4) outside $\mathcal{P}_{\lambda} \subseteq \mathcal{S}$. In particular, $\bar{N}\left[\Psi_{\mathcal{P}_{\lambda}}, 1\right] \subset \mathcal{S}$, and for $x \notin \bar{N}\left[\Psi_{\mathcal{P}_{\lambda}}, 1\right], \mathcal{D}^{+} \Psi_{\mathcal{P}_{\lambda}}(x(t)) \leq \frac{\lambda-1}{\tau} \Psi_{\mathcal{P}_{\lambda}}(x(t))$.

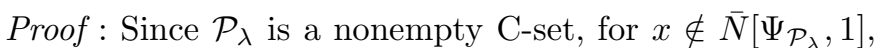
there exist a $\mu \geq 1$ such that $x$ lies on the boundary of of set $\bar{N}\left[\Psi_{\mathcal{P}_{\lambda}}, \mu\right]$, i.e., $x \in \partial \bar{N}\left[\Psi_{\mathcal{P}_{\lambda}}, \mu\right]$. In addition, $\mathcal{P}_{\lambda}$ is $\lambda$ expansive with respect to system (8), so is $\mu \mathcal{P}_{\lambda}$ for $\mu \geq 1$. Therefore, for $x \in \partial \bar{N}\left[\Psi_{\mathcal{P}_{\lambda}}, \mu\right], \operatorname{post}_{q}(x, \mathcal{W}, \mathcal{D}) \subseteq \lambda \mu \mathcal{P}_{\lambda}$.
Hence, for $w \in \mathcal{W}, d \in \mathcal{D}, \Psi_{\mathcal{P}_{\lambda}}([I+\tau A(w)] x+\tau E d) \leq$ $\lambda \Psi_{\mathcal{P}_{\lambda}}=\lambda \mu$ holds for some $\tau>0$. So,

$$
\frac{\Psi_{\mathcal{P}_{\lambda}}\left(\left[I+\tau A_{q}(w)\right] x+\tau E_{q} d\right)-\Psi_{\mathcal{P}_{\lambda}}(x)}{\tau} \leq \frac{\lambda-1}{\tau} \Psi_{\mathcal{P}_{\lambda}}(x)
$$

holds for some $\tau>0$. Since $\Psi_{\mathcal{P}_{\lambda}}(x)$ is a convex function, and $\Psi_{\mathcal{P}_{\lambda}}(x)=\mu$ is finite, then the difference quotient is a nondecreasing function for $\tau$ (Rockafellar [1972], Section 23). In addition, $\mathcal{D}^{+} \Psi_{\mathcal{P}_{\lambda}}(x(t))$ exists and

$$
\begin{aligned}
\mathcal{D}^{+} \Psi_{\mathcal{P}_{\lambda}}(x(t)) & =\inf _{\tau>0} \frac{\Psi_{\mathcal{P}_{\lambda}}\left(x+\tau\left[A_{q}(w) x+E_{q} d\right]\right)-\Psi_{\mathcal{P}_{\lambda}}(x)}{\tau} \\
& \leq \frac{\lambda-1}{\tau} \Psi_{\mathcal{P}_{\lambda}}(x)
\end{aligned}
$$

Since this inequality holds for any $w \in \mathcal{W}$ and $d \in \mathcal{D}$, the Dini derivative

$$
\mathcal{D}^{+} \Psi_{\mathcal{P}_{\lambda}}(x(t)) \leq \frac{\lambda-1}{\tau} \Psi_{\mathcal{P}_{\lambda}}(x(t))
$$

at point $x(t)=x$. Since $x$ is arbitrary point outside $\mathcal{P}_{\lambda}$, we conclude that the function $\Psi_{\mathcal{P}_{\lambda}}(x)$ is a Lyapunov-like function for system (1) outside $\mathcal{P}_{\lambda} \subseteq \mathcal{S}$.

\subsection{UUB Analysis}

Based on similar techniques in Blanchini [1995], it can be argued that it does not cost generality to focus only on the polyhedral Lyapunov-like functions under the existence assumption of a Lyapunov-like function for (4). Therefore, it is assumed that each unstable subsystem has an expansive index $\lambda_{q}>1$ to $\mathcal{S}$ along with a polyhedral Lyapunov-like function, $\Psi_{q}(\cdot)$. Similarly, define the multiple Lyapunov function candidate as

$$
V(x(t))=\Psi_{\sigma(t)}(x(t))
$$

For any switching signal $\sigma(t)$ and any $t>0$, let $K_{i}(t)$ denote the total period that the $q_{i}$-th subsystem is activated during $[0, t)$. Define $K^{-}(t)=\sum_{i \leq r, q_{i} \in Q} K_{i}(t)$, which stands for the total activation period of the UUB subsystems. On the other hand, $K^{+}(t)=\sum_{i>r, q_{i} \in Q} K_{i}(t)$ denotes the total activation period of the non-UUB subsystems. We have $K^{-}(t)+K^{+}(t)=t$.

For any $t$ satisfying $t_{0}=0<\cdots<t_{i} \leq t<t_{i+1}$, we obtain

$$
V(x(t)) \leq \begin{cases}\max \left\{e^{-\beta_{q_{i}}\left(t-t_{i}\right)} \Psi_{q_{i}}\left(x\left(t_{i}\right)\right), 1\right\} & q_{i} \leq r \\ \max \left\{e^{\beta_{q_{i}}\left(t-t_{i}\right)} \Psi_{q_{i}}\left(x\left(t_{i}\right)\right), 1\right\} & r<q_{i} \leq N\end{cases}
$$

Let us define $\beta_{s}=\min _{1 \leq i \leq r}\left\{\beta_{q_{i}}\right\}$, and $\beta_{u}=\max _{r<i \leq N}\left\{\beta_{q_{i}}\right\}$. Also, there exists a constant scalar $\mu$ such that $\Psi_{q_{i}}(x) \leq$ $\mu \Psi_{q_{j}}(x)$ and $\Psi_{q_{j}}(x) \leq \mu \Psi_{q_{i}}(x)$, for all $x \in \mathbb{R}^{n}$. The scalar $\mu$ can be selected as in the previous section.

Therefore, by induction, we have

$$
V(x(t)) \leq \max \left\{e^{-\beta_{s} K^{-}(t)+\beta_{u} K^{+}(t)} \mu^{N_{\sigma}(t)} V(x(0)), 1\right\}
$$

If there exists a positive scalar $\beta<\beta_{s}$ such that

$$
\frac{K^{+}(t)}{t} \leq \frac{\beta_{s}-\beta}{\beta_{u}+\beta_{s}}
$$

which is a condition on the percentage of time interval that the unstable subsystems are activated, then we obtain 


$$
e^{-\beta_{s} K^{-}(t)+\beta_{u} K^{+}(t)} \leq e^{-\beta t}
$$

And thus

$$
V(x(t)) \leq \max \left\{e^{-\beta t} \mu^{N_{\sigma}(t)} V(x(0)), 1\right\} .
$$

Also assume that

$$
N_{\sigma}(t) \leq \frac{t}{\tau_{a}^{*}}, \quad \tau_{a}^{*}=\frac{\ln \mu}{\beta-\beta^{*}}
$$

for some positive scalar $\beta^{*}<\beta$, which represent a bounded average dwell time requirement. This implies

So,

$$
e^{-\beta t} \mu^{N_{\sigma}(t)} \leq e^{-\beta^{*} t}
$$

$V(x(t)) \leq \max \left\{e^{-\beta^{*} t} V(x(0)), 1\right\}$.

This implies that the entire system is UUB.

Theorem 14. The switched system (2) achieves a finite asymptotic disturbance attenuation level under switching signals

(1) with percentage of time interval that the unstable subsystems are activated less than $\frac{\beta_{s}-\beta}{\beta_{u}+\beta_{s}}$ in the sense of (14), and

(2) with average dwell time no less than $\frac{\ln \mu}{\beta-\beta^{*}}$ in the sense of (16).

Compared with the result for all subsystems are UUB, the switching signals in this section have one condition on the percentage of the activation periods of unstable subsystems in addition to a similar average dwell time condition. In other words, the switched system that stays too long in the unstable mode may lead to an unbounded disturbance attenuation performance.

\section{CONCLUDING REMARKS}

In this paper, we investigated the asymptotic disturbance attenuation properties for a class of switched linear systems with parametric uncertainties and exterior disturbances under various switching signals. First, arbitrary switching signals were considered, and a necessary and sufficient condition on the subsystems' dynamics was derived for the switched systems to achieve a finite disturbance attenuation level under the assumption that each subsystem was UUB. In addition, the equivalence between the asymptotic stability of arbitrary switching linear systems and the robust stability of a corresponding linear timevariant systems was obtained, which bridges two originally distinct research fields. Secondly, if the finite disturbance attenuation level is not preserved under arbitrary switch signals, average dwell switching schemes were proposed to attain a finite disturbance attenuation level. Two cases were considered for this problem that depend on whether all the subsystems are UUB or not. The techniques were based on multiple set-induced Lyapunov functions and polyhedral algebra. Another contribution of this paper is the extension of set-induced Lyapunov-like function for unstable systems, which is useful to characterize the explosiveness of an unstable systems.

\section{REFERENCES}

F. Blanchini. Ultimate boundedness control for uncertain discrete-time systems via set-induced Lyapunov func- tions. IEEE Trans. Automat. Contr., vol. 39, no. 2, pp. 428-433, 1994.

F. Blanchini. Nonquadratic Lyapunov functions for robust control. Automatica, vol. 31, pp. 451-461, 1995.

R. K. Brayton, C. H. Tong. Stability of dynamic systems: a constructive approach. IEEE Trans Circ Sys vol. 26, no. 4, pp. 224-23, 1979.

W. Dayawansa and C. F. Martin. A converse Lyapunov theorem for a class of dynamical systems which undergo switching. IEEE Trans. Automat. Contr., vol. 44, no. 4, pp. 751-760, 1999.

R. A. Decarlo, M. S. Branicky, S. Pettersson, and B. Lennartson. Perspectives and results on the stability and stabilizability of hybrid systems. Proccedings of the IEEE, vol. 88, no. 7, pp. 1069-1082, 2000.

J. P. Hespanha and A. S. Morse. Stability of switched systems with average dwell-time. in Proceedings of the 38th IEEE Conf. Decision Control, pp. 2655-2660, 1999.

J. P. Hespanha and A. S. Morse. Switching between stabilizing controllers. Automatica, vol. 30, no. 11, pp. 1905-1917, 2002.

J. P. Hespanha. Root-mean-square gains of switched linear systems. IEEE Trans. Automat. Contr., vol. 48, no. 11, pp. 2040-2045, 2003.

J. P. Hespanha. Uniform stability of switched linear systems: Extensions of LaSalle's invariance principle. IEEE Trans. Automat. Contr., vol. 49, no. 4, pp. 470482, 2004.

D. Liberzon and A. S. Morse. Basic problems in stability and design of switched systems. IEEE Contr. Syst. Magazine, vol. 19, no. 15, pp. 59-70, 1999.

D. Liberzon. Switching in Systems and Control. Birkhauser, Boston, 2003.

H. Lin and P. J. Antsaklis. Stability and stabilizability of switched linear systems: A short survey of recent results. in Proc. of 2005 ISIC-MED Joint Conference, pp. 24-29, 2005.

H. Lin and P. J. Antsaklis. Switching stabilizability for continuous-time uncertain switched linear systems. IEEE Trans. Automat. Contr., vol. 52, no. 4, pp. 633646, 2007.

A. P. Molchanov and E. Pyatnitskiy. Criteria of asymptotic stability of differential and difference inclusions encountered in control theory. Systems \& Control Letters, vol. 13, pp. 59-64, 1989.

A. Polański. On absolute stability analysis by polyhedral Lyapunov functions. Automatica, vol. 36, no. 4, pp. 573-578, 2000.

R.T. Rockafellar. Convex Analysis. Princeton University Press, Princeton, NJ. 1972.

Z. Sun and S. S. Ge. Analysis and synthesis of switched linear control systems. Automatica, vol. 40, no. 2, pp. 181-195, 2005.

C. Yfoulis and R. Shorten. A numerical technique for stability analysis of linear switched systems. Inter. J. Contr. vol. 77, no. 11, pp. 1019-1039, 2004.

G. Zhai, B. Hu, K. Yasuda, and A. N. Michel. Disturbance attenuation properties of time-controlled switched systems. J. Franklin Institute, vol. 338, pp. 765-779, 2001.

G. Zhai, B. Hu, K. Yasuda, and A. N. Michel. Qualitative analysis of discrete-time switched systems. in Proc. 2002 American Control Conf., pp. 1880-1885, 2002. 